\title{
Contornos y perfil de emergencia: aplicación clínica e importancia en la terapia restauradora
}

\section{Contours and emergence profile: clinical application and importance in the restorative therapy}

\author{
Gómez Mira F*, Ardila Medina CM**
}

\section{RESUMEN}

Una adecuada atención de los tejidos duros y blandos alrededor del diente y del implante antes, durante y después de los procedimientos restauradores incrementarán enormemente la probabilidad de un buen resultado. Los contornos coronales son determinados normalmente por la anatomía dentaria, la condición periodontal, la localización del margen gingival de la restauración y el acceso a la higiene bucal, sin dejar de considerar el compromiso estético subyacente. Los contornos de una restauración apropiada requieren una reducción adecuada del pilar que permita un espesor propicio de los materiales restauradores, mientras se permite a la vez un acceso fácil a la higiene dental. El perfil de emergencia de una restauración en un área estética presenta dos aspectos: la forma subgingival y la forma supragingival. La forma subgingival debe seguir los contornos de la unión cemento esmalte y el soporte de los tejidos gingivales. Considerando las limitaciones, el espesor aumentado de los contornos subgingivales interproximales conduce a un incremento en la altura de la papila, mientras que una ampliación de los contornos faciales favorece un posicionamiento apical de los tejidos gingivales. Estos y otros aspectos clínicos deben ser conocidos por el odontólogo cuando desea realizar una restauración con éxito.

Palabras clave: Contornos, perfil de emergencia, restauración.

\section{SUMMARY}

Close attention to both soft and hard tissues around teeth and implants before, during, and after restorative procedures will greatly increase the probability of a successful outcome. Crown contours are normally determined by tooth anatomy, periodontal condition, margin placement, and access for oral hygiene. However, compromises must occasionally be made in the interest of aesthetics. Proper restorative contours require adequate tooth reduction to allow proper thickness of restorative materials, while allowing easy access for personal oral hygiene. The emergence profile of a restoration in aesthetic areas has two aspects: subgingival form and supragingival form. The subgingival form should follow the contours of the cementoenamel junction and support the gingival tissues. Within limits, increased thickness of interproximal subgingival contours leads to increased papillary height, while increased facial contours lead to apical positioning of the gingival tissues. These and other clinical aspects must be known by the dentist when wishes to make a successful restoration.

Key words: Contours, emergence profile emergency, restoration.

Fecha de recepción: 7 de noviembre de 2008.

Aceptado para publicación: 11 de noviembre de 2008.

* $\quad$ Rehabilitador Oral. Universidad de Antioquía.

** Profesor Asistente. Universidad de Antioquía. Presidente Sdad. Colombiana de Periodoncia-Regional Antioquía.

Gómez Mira F, Ardila Medina CM. Contornos y perfil de emergencia: aplicación clínica e importancia en la terapia restauradora. Av. Odontoestomatol 2009; 25 (6): 331-338. 


\section{INTRODUCCIÓN}

Se conoce que el efecto de la odontología restauradora sobre la salud del periodonto está influenciado por las propiedades físicas y químicas de los materiales empleados (1), el ajuste marginal (2), la posición del margen coronal (3), el contorno coronal (4, $5)$, el perfil de emergencia $(6,7)$, y la oclusión de la restauración (8). Existen opiniones controvertidas cuando se considera la influencia de los contornos coronales artificiales (9). Es así como diferentes autores han mencionado que desde el punto de vista periodontal, se prefieren las coronas infracontorneadas $(10,11)$. Sin embargo, en pacientes periodontalmente comprometidos, es obligado ubicar márgenes coronales subgingivales y sobrecontornearlos por razones estéticas (12). Por otra parte, estudios clínicos han presentado el impacto de diferentes perfiles de emergencia sobre la calidad y cantidad de la placa bacteriana $(13,14)$. Algunas teorías han tratado de explicar la necesidad de formas particulares de los contornos axiales de coronas parciales y completas, argumentándose que muchas ideas relacionadas con los contornos sugeridos para restauraciones no están basados en evidencia científica (15).

El objetivo de este artículo es presentar aspectos clínicos fundamentales de los contornos coronales y del perfil de emergencia que deben considerarse para la realización de una restauración con éxito, con base en la evidencia disponible actualmente.

\section{CONTORNOS CORONALES}

Una restauración que sigue la anatomía natural de los contornos del diente puede mantener y estimular funcionalmente la salud de los tejidos gingivales (15, 16) (Fig. 1). El Glosario de Términos Prostodóncicos define contorno como el perfil de la curva del diente o la línea que representa este perfil (17). Varios estudios han descrito las características de los contornos dentales y sus implicaciones protésico periodontales $(18,19)$, observándose como las lesiones dentales ocasionan con frecuencia cambios en los contornos coronales, como ocurre en los casos de dientes fracturados o desgastados, creando disturbios visuales que influyen sobre la apariencia general de las estructuras bucales (4). Igualmente, diferentes autores

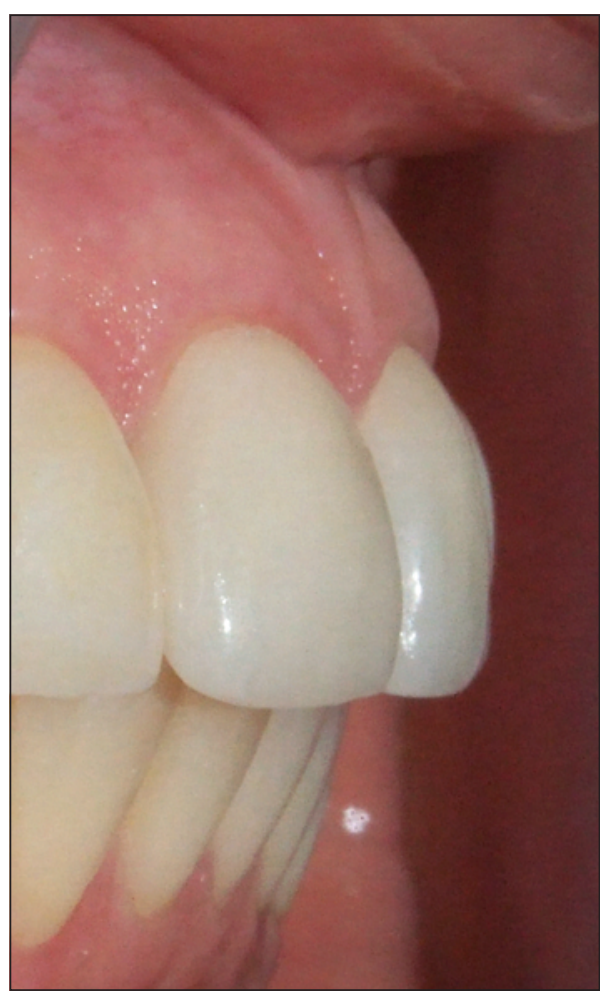

Fig. 1.

Restauración que sigue la anatomía natural de los contornos del diente.

han demostrado que la retención de placa sobre las superficies bucales y linguales se presenta en primer lugar debajo de la protuberancia dental supragingival $(9,20)$ Se ha sugerido que el sobrecontorno de las restauraciones protésicas es uno de los factores iatrogénicos que conducen a la acumulación de placa bacteriana sobre las coronas, ocasionando inflamación y sangrado de los tejidos periodontales, especialmente en las superficies proximales $(9,21)$ (Fig. 2). Hasta la fecha, las investigaciones sobre morfología coronal dental se han realizado mediante estudios descriptivos y evaluaciones cuantitativas empleando observaciones visuales $(18,19,22-25)$, enfoque fotográfico (26), medidas clínicas con calibradores (27) y análisis matemáticos y computarizados $(4,28)$. Sin embargo, aún continúa siendo un desafío capturar datos morfológicos dentales de tal forma que permitan comparaciones cuantitativas rigurosas (29-31).

A continuación se presentan algunas investigaciones que han tratado de dilucidar la importancia de los contornos coronales en la odontología restauradora y en el mantenimiento saludable de los tejidos gingivales. 


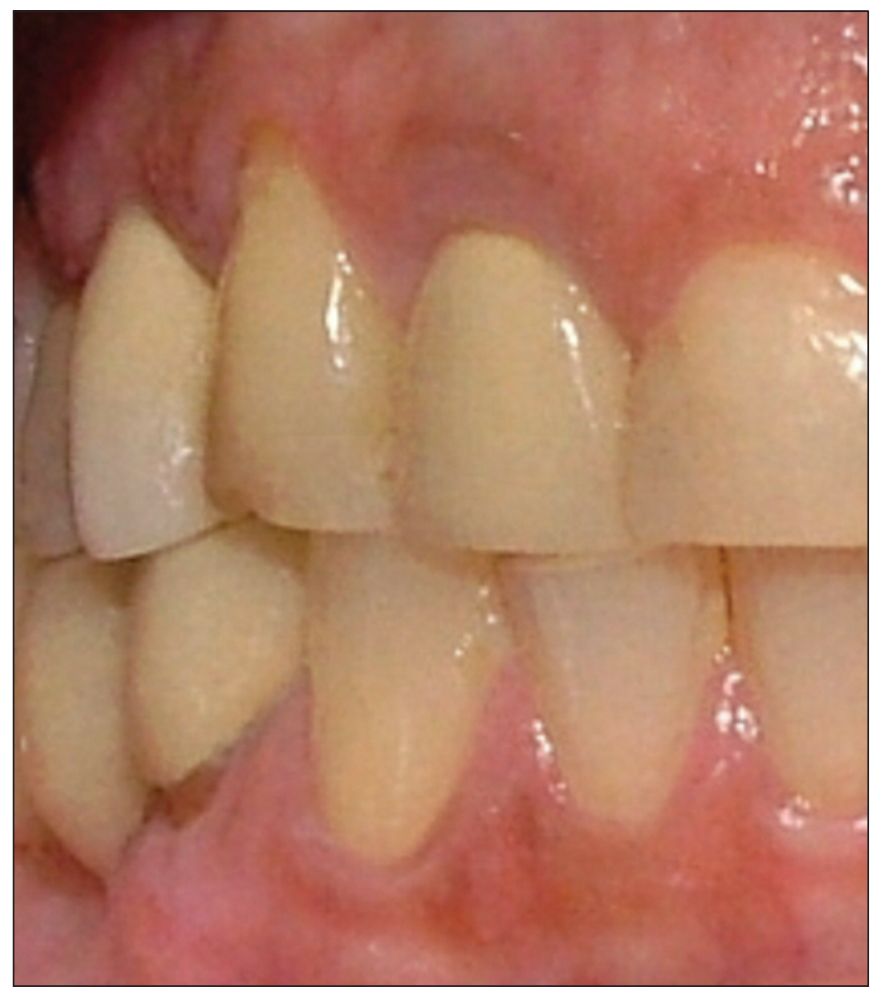

Fig. 2. Restauraciones protésicas que favorecen la inflamación gingival.

Goodacre y colaboradores (19), revisaron los artículos más relevantes relacionados con el conocimiento científico sobre preparaciones dentarias para coronas completas en los últimos 50 años, indicando que la salud periodontal puede mantenerse en presencia de márgenes intracreviculares, lo cual requiere restauraciones adecuadamente contorneadas con márgenes satisfactorios y tratamiento cuidadoso de los tejidos duros y blandos asociados con la preparación dentaria.

Con el fin de investigar la relación del contorno coronal axial y la salud de los tejidos gingivales circundantes (24), se realizaron tres cortes a través de la corona y la encía de premolares mandibulares en modelos dentales, encontrando una relación única entre la corona axial y el contorno gingival. Esta característica del diente es similar a su diente contralateral de tal manera que es una guía fundamental cuando se reemplaza un diente perdido.

La necesidad de reconstruir una réplica gingival en la reproducción del contorno de la encía, en una restauración protésica sobre un diente o implante, llevó a proponer una técnica para duplicar el surco natural y la morfología de la encía libre con materiales tipo silicona (25). Concluyen los autores que de esta forma se asegura una adecuada reconstrucción de la morfología y del contorno coronal compatible con estética y salud gingival.

Con el fin de preservar el contorno de los tejidos blandos y duros existentes e igualmente obtener buenos resultados estéticos, se han propuesto diferentes técnicas para realizar restauraciones provisionales sobre implantes $(4,7,8)$ (Figs. 3 y 4). Es así

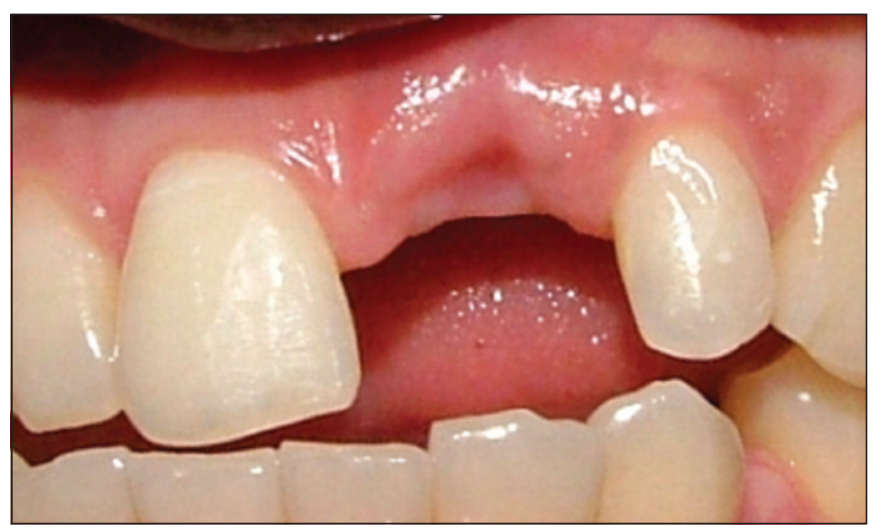

Fig. 3. Espacio edéntulo que va a ser restaurado con implante de carga inmediata.

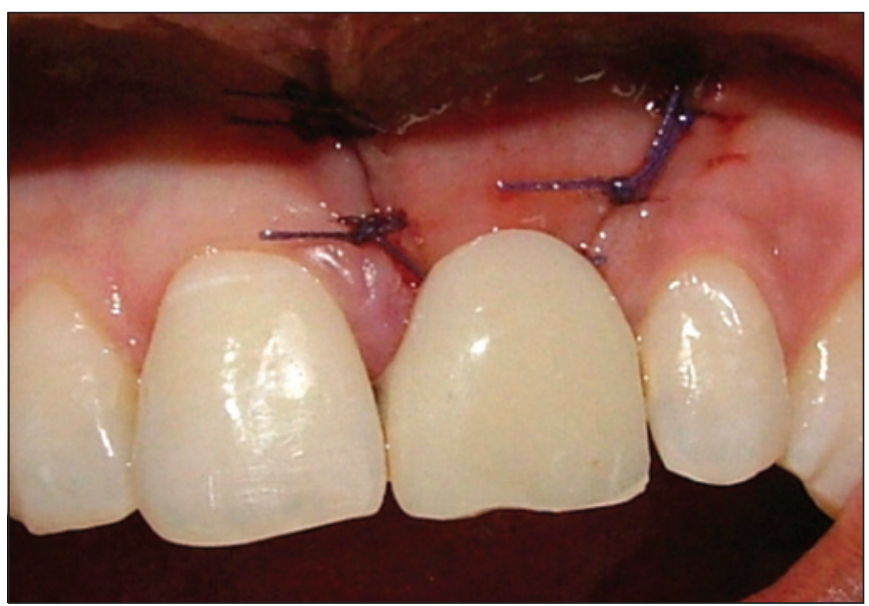

Fig. 4. Restauración provisional sobre implante que conserva contornos y perfil de emergencia adecuados. 
como Al Harbi y colaboradores (5), describieron la fabricación de un provisional que según ellos, reunió todos los requisitos anatómicos, biológicos y estéticos, en donde el conocimiento adecuado de los contornos coronales y su relación con los tejidos blandos circundantes, fue fundamental para conseguir resultados exitosos.

Las alteraciones dimensionales de los tejidos periimplantarios en restauraciones individuales fue evaluada en un estudio prospectivo que incluyó 11 pacientes entre los 18 y 36 años (32). Los autores encontraron que cuando no se tuvieron presentes los conceptos básicos en cuanto a contornos coronales se refiere y a su relación con los tejidos gingivales, se observó inicialmente un incremento del volumen bucal después de la colocación de la corona, seguido por un desplazamiento apical del margen gingival de aproximadamente $0.6 \mathrm{~mm}$ en promedio después de un año, con implicaciones estéticas graves.

Usando una aplicación de análisis de imágenes mediante programas computarizados (28), se determinó sí 55 coronas completas estaban sobrecontorneadas comparando su contorno en el plano medio buco lingual, con su equivalente diente natural, en el lado opuesto del mismo arco. Se encontró que la mayoría de las coronas presentaban sobrecontornos, especialmente en la superficie lingual.

Yu y colaboradores (4), evaluaron 116 voluntarios chinos con el fin de desarrollar un método para analizar el contorno de los dientes y de las restauraciones. En cada paciente se fabricó un modelo superior con resina acrílica autocurada, en donde se obtuvieron imágenes seriales de cortes de los dientes, con el fin de obtener un grupo de datos para ser analizados y así encontrar ecuaciones apropiadas que describieran la forma de los contornos. Las superficies bucales presentaron un contorno coronal mas recto en los hombres y se volvieron gradualmente más rectas desde el canino al incisivo central, encontrándose la mayor convexidad en el tercio mesial de cada diente. En la superficie lingual, la mayor concavidad se encontró en el tercio medio de cada diente, siendo superior en el canino.

Diferentes estudios han descrito las características de los contornos de acuerdo a la posición de los dientes en el arco dental, mencionando los siguientes hallazgos (Tabla 1): las crestas de los contornos bucales de todos los dientes se encuentran en el tercio gingival de la corona, con una proyección vestibular de hasta $0.5 \mathrm{~mm}$ a partir de la unión cemento esmalte (33). Entre la superficie oclusal o incisal y la cresta bucal, la superficie vestibular presenta una leve convexidad. La superficie bucal de los dientes posteriores inferiores es convexa entre las crestas gingivales y las superficies oclusales. Existen leves depresiones bucales entre la superficie oclusal/incisal y la cresta gingival en dirección vertical (34). De manera igual, la mayor convexidad de las superficies linguales de algunos dientes se encuentra en el tercio gingival, pero en los molares y premolares inferiores, la convexidad lingual se encuentra en el tercio medio. Se considera que la convexidad lingual debe protruir lingualmente solo $0,5 \mathrm{~mm}$ a partir de la unión cemento esmalte en cualquier diente, excepto en los premolares y molares inferiores, donde la convexi-

\section{TABLA 1.- CARACTERÍSTICAS DE LOS CONTORNOS CORONALES DE ACUERDO A LA POSICIÓN DE LOS DIENTES EN EL ARCO DENTAL (33-35)}

- Los contornos bucales de todos los dientes se encuentran en el tercio gingival de la corona.

- Entre la superficie oclusal o incisal y la cresta bucal, la superficie vestibular presenta una leve convexidad.

- La superficie bucal de los dientes posteriores inferiores es convexa entre las crestas gingivales y las superficies oclusales.

- Existen leves depresiones bucales entre la superficie oclusal/incisal y la cresta gingival en dirección vertical.

- La mayor convexidad de las superficies linguales de algunos dientes se encuentra en el tercio gingival.

- La convexidad lingual debe protruir lingualmente solo $0,5 \mathrm{~mm}$ a partir de la unión cemento esmalte en cualquier diente, excepto en los premolares y molares inferiores.

- Los dos tercios cervicales de las superficies proximales son planos o ligeramente cóncavos. 
dad máxima puede extenderse hasta $0,75-1 \mathrm{~mm}$ $(34,35)$. Se han descrito los dos tercios cervicales de las superficies proximales como planos o ligeramente cóncavos. El diente es plano o levemente cóncavo desde la superficie bucal a la lingual, también como desde el nivel del contacto proximal hasta la unión cemento esmalte. La excepción es la superficie distal del primer molar superior, el cual es de forma convexa, pero cervicalmente existe una concavidad $(34,35)$.

\section{PERFIL DE EMERGENCIA}

Comprender la anatomía dental y el complejo dentogingival ha permitido el establecimiento del concepto de perfil de emergencia (PE), término que ha tenido distintos significados. En 1989, se definió como la porción del contorno dental axial que se extiende desde la base del surco gingival hasta el margen libre de la encía (26). El Glosario de Términos Prostodóncicos lo define como el contorno de un diente o una restauración, ya sea una corona sobre un diente natural o un pilar de un implante, y su relación con los tejidos adyacentes (17). Pocos artículos establecen que el PE sea recto (36), plano o cóncavo $(37,38)$. Aún cuando se han observado algunas concavidades y convexidades, la norma es un $P E$ recto (39). El PE es el nexo más crucial entre la forma del diente y la salud gingival. Se requiere una atención muy cuidadosa cuando se establezca un PE adecuado en la restauración definitiva para que se reduzcan no solamente las áreas retentivas que permitan la acumulación de placa bacteriana sino también la inflamación iatrogénica $(40,41)$. Varias técnicas se han diseñado para ayudar a los clínicos y a los técnicos dentales a crear el correcto PE en las restauraciones definitivas (42-46). Ello incluye observación de los tejidos blandos $(45,47)$, réplicas gingivales (25) y restauraciones provisionales $(44,46,47)$.

La mayoría de los artículos relacionados con el estudio del PE tratan sobre distintas técnicas de laboratorio, restauraciones provisionales y aspectos teóricos a tener en cuenta con el fin de conseguir tratamientos exitosos protésica y periodontalmente, pero son pocos los estudios clínicos que muestran las implicaciones de distintos PE en las poblaciones, fundamentalmente por principios éticos.
Croll (39), asegura que la selección de un PE recto mejora la efectividad de la higiene oral cerca del surco gingival, cuando se diseñan coronas artificiales sobre dientes naturales. Así mismo, empleando datos fotográficos (26), confirmó que en los dientes naturales el PE es recto y que es esencial su reproducción utilizando modelos anatómicos confiables.

El efecto de diferentes PE sobre el periodonto fue evaluado clínica y microbiológicamente $(9,21)$. Un mes después de iniciar procedimientos de higiene oral, el segundo y tercer premolar de tres cuadrantes de cuatro perros beagle fueron preparados para recibir coronas completas empleando tres diferentes tipos de PE: contorno normal, sobrecontornos de $30^{\circ}$ y $50^{\circ}$. Se observó mayor cantidad de placa bacteriana e inflamación gingival, incremento de la profundidad de sondaje y mayor pérdida de inserción en los dientes sobrecontorneados, a pesar de que se implementaron regímenes exigentes de higiene oral. La frecuencia de detección de algunas especies bacterianas fue mayor en las coronas sobrecontorneadas, comparadas con las coronas con contorno normal y con los dientes naturales.

Se evaluaron 50 dientes anteriores con coronas completas y sus dientes homólogos contra laterales con el fin de encontrar valores geométricos de los PE y estudiar su influencia sobre el estado periodontal (6). Los parámetros clínicos estudiados fueron el índice de placa bacteriana (48), el índice gingival (49), la profundidad de sondaje y el nivel de inserción clínica. Se realizaron impresiones y modelos de yeso, los cuales fueron seccionados a lo largo de la línea media de cada diente. Todos los especímenes fueron escaneados y transferidos en una forma gráfica con un programa computarizado, procesando y registrando los datos de los PE de los dientes naturales y restaurados. El promedio del PE supragingival para las superficies bucales y linguales de los dientes naturales fueron $11,13 \pm 7,92$ y $15,8 \pm 9,16$ grados, respectivamente. Los valores correspondientes para los $\mathrm{PE}$ subgingivales fueron 9,93 $\pm 5,68$ y 14,35 $\pm 8,44$ grados (Tabla 2). Los parámetros periodontales fueron mayores en los dientes restaurados que en los dientes naturales. Cuando el índice de placa y el género fueron controlados, se encontró una correlación entre el PE subgingival lingual de los dientes restaurados, la profundidad de sondaje y la pérdida de inserción, 
TABLA 2.- PERFILES DE EMERGENCIA PROMEDIO EN DIENTES NATURALES (6)

\begin{tabular}{|l|c|c|}
\hline & Supragingival & Subgingival \\
\hline Superficies bucales & $11,13 \pm 7,92^{\circ}$ & $9,93 \pm 5,68^{\circ}$ \\
Superficies linguales & $15,8 \pm 9,16^{\circ}$ & $14,35 \pm 8,44^{\circ}$ \\
\hline
\end{tabular}

${ }^{\circ}$ : grados.

concluyendo que el PE de los dientes restaurados pueden afectar el estado periodontal, especialmente en la superficie lingual.

Con el propósito de evaluar el efecto de las coronas con diferentes PE sobre la formación de placa marginal se realizaron siete coronas completas sobre premolares en seis pacientes (14). Aquellas coronas de titanio y con PE de hasta $40^{\circ}$, presentaron menor acumulación de placa bacteriana que los pacientes controles. Es importante aclarar que los resultados de este estudio deben ser analizados con cautela debido al pequeño tamaño muestral.

\section{CONCLUSIONES}

El índice de placa (48), el índice gingival (49), la profundidad de sondaje y el nivel de inserción son mayores en los dientes restaurados, indicando que una adecuada higiene oral y una salud periodontal óptima es más difícil de mantener en presencia de restauraciones.

Los márgenes subgingivales sobrecontorneados y PE exagerados pueden alterar la estética e integridad de los tejidos gingivales y se encuentran más asociados a inflamación y retención de placa bacteriana.

Los contornos y PE modificados por las restauraciones pueden no ser el único factor responsable del consecuente cambio del estado periodontal, especialmente en la superficie lingual, la cual puede verse afectada por la visión indirecta empleada allí cuando se realizan las preparaciones protésicas, la dificultad inherente del lugar durante la impresión y el acceso dificultoso a la higiene bucal en esa superficie.

Contornos adecuados y PE rectos son aceptados como estrategias para simplificar la higiene bucal.
La mayoría de los estudios muestran que son más lesivos para la salud gingival los sobrecontornos que los infracontornos.

Una buena recomendación cuando se restaura un diente es reconstruirlo y evaluarlos estableciendo una comparación con el diente natural contralateral.

La restauración temporal es un paso crucial para la evaluación de los parámetros estéticos, protésicos y periodontales.

\section{BIBLIOGRAFÍA}

1. Schmalz G, Garhammer P. Biological interactions of dental cast alloys with oral tissues. Dent Mater 2002; 18:396-406.

2. Carr AB. Effect of prosthetic remedial treatments on the oral health status of individuals and populations. Int J Prosthodont 2003;16 Suppl: 55-8.

3. Kancyper SG, Koka S. The influence of intracrevicular crown margins on gingival health: preliminary findings. J Prosthet Dent 2001;85: 461-5.

4. Yu H, Li Q, Hu J, Wang Y. An improved method to analyse tooth and restoration contour using image analysis: application in the maxillary anterior teeth in Chinese population. Arch Oral Biol 2008;53:503-8.

5. Al-Harbi SA, Edgin WA. Preservation of soft tissue contours with immediate screw-retained provisional implant crown. J Prosthet Dent 2007;98: 329-32.

6. Yotnuengnit B, Yotnuengnit $\mathrm{P}$, Laohapand $\mathrm{P}$, Athipanyakom S. Emergence angles in natural anterior teeth: influence on periodontal status. Quintessence Int. 2008;39:126-33.

7. Macintosh DC, Sutherland M. Method for developing an optimal emergence profile using heat-polymerized provisional restorations for single-tooth implant-supported restorations. J Prosthet Dent 2004;91:289-92.

8. Cordaro L, Ercoli C, Rossini C, Torsello F, Feng C. Retrospective evaluation of complete-arch 
fixed partial dentures connecting teeth and implant abutments in patients with normal and reduced periodontal support. J Prosthet Dent 2005;94:313-20.

9. Kohal RJ, Gerds T, Strub JR. Effect of different crown contours on periodontal health in dogs. Clinical results. J Dent. 2003;31:407-13.

10. Näpänkangas R, Salonen MA, Raustia AM. A 10year follow-up study of fixed metal ceramic prosthodontics. J Oral Rehabil 1997;24:713-7.

11. Kancyper SG, Koka S. The influence of intracrevicular crown margins on gingival health: preliminary findings. J Prosthet Dent 2001;85: 461-5.

12. Jørnung J, Fardal $\varnothing$. Perceptions of patients' smiles: a comparison of patients' and dentists' opinions. J Am Dent Assoc 2007;138:1544-53.

13. Konradsson K, Claesson R, van Dijken JW. Mutans streptococci and lactobacilli in plaque on a leucitereinforced dental ceramic and on a calcium aluminate cement. Clin Oral Investig 2006;0:175-80.

14. Sundh B, Köhler B. An in vivo study of the impact of different emergence profiles of procera titanium crowns on quantity and quality of plaque. Int J Prosthodont. 2002;15:457-60.

15. Becker CM, Kaldahl WB. Current theories of crown contour, margin placement, and pontic design. 1981. J Prosthet Dent 2005;93:107-15.

16. Crespi R, Grossi SG. The emergence margin in prosthetic reconstruction of periodontally involved teeth. Int J Periodontics Restorative Dent 1993; 13:348-59.

17. Academy of Prosthodontics The glossary of prosthodontic terms. J Prosthet Dent. 2005;94:10-92.

18. Goodacre CJ. Designing tooth preparations for optimal success. Dent Clin North Am 2004;48: 359-85.

19. Goodacre CJ, Campagni WV, Aquilino SA. Tooth preparations for complete crowns: an art form based on scientific principles. J Prosthet Dent. 2001;85:363-76.

20. Koke U, Sander C, Heinecke A, Müller HP. A possible influence of gingival dimensions on attachment loss and gingival recession following placement of artificial crowns. Int J Periodontics Restorative Dent 2003;23:439-45.

21. Kohal RJ, Pelz K, Strub JR. Effect of different crown contours on periodontal health in dogs. Microbiological results. J Dent 2004;32:153-9.

22. Burch JG, Miller JB. Evaluating crown contours of a wax pattern. J Prosthet Dent 1973;30: 454-8.

23. Silverstein LH, Kurtzman GM, Kurtzman D, Shatz PC, Szikman R. Placing dental implants and/or natural tooth restorations in the aesthetic zone: achieving proper gingival contours. Dent Today 2007;26:132-5.

24. Wu Y, Xu J.Geometric relationship of axial crown contour and gingiva contour of normal second mandibular premolars. Zhonghua Kou Qiang Yi Xue Za Zhi 2000;35:274-6.

25. Bassiouny MA, Yearwood LL. Establishing the gingival emergence profile of restorations by using a resilient gingival replica. J Prosthet Dent 1996;76:386-9.

26. Croll BM. Emergence profiles in natural tooth contour. Part 1: photographic observations. J Prosthet Dent 1989; 62:4-10.

27. Davis MV. The importance of contour on full coverage restorations. Pract Periodontics Aesthet Dent 1992;4:17-23.

28. Alhouri N, Watts DC, McCord JF, Smith PW. Mathematical analysis of tooth and restoration contour using image analysis. Dent Mater 2004; 20:893-9.

29. Robinson DL, Blackwell PG, Stillman EC, Brook AH. Planar Procrustes analysis of tooth shape. Arch Oral Biol 2001;46:191-9. 
30. Robinson DL, Blackwell PG, Stillman EC, Brook $\mathrm{AH}$. Impact of landmark reliability on the planar Procrustes analysis of tooth shape. Arch Oral Biol. 2002;47:545-54.

31. Martinón-Torres M, Bastir M, Bermúdez de Castro JM, Gómez A, Sarmiento S, Muela A, et al. Hominin lower second premolar morphology: evolutionary inferences through geometric morphometric analysis. J Hum Evol 2006;50: 523-33.

32. Cardaropoli G, Lekholm U, Wennström JL.Tissue alterations at implant-supported single-tooth replacements: a 1-year prospective clinical study. Clin Oral Implants Res 2006;17:165-71.

33. Wheeler RC. Complete crown form and the periodontium. J Prosthet Dent 1961;11:722-34.

34. Koidis PT, Burch JG, Melfi RC. Clinical crown contours: contemporary view. J Am Dent Assoc 1987;114:792-5.

35. Burch JG. Ten rules for developing crown contours in restorations. Dent Clin North Am 1971;15:611-8.

36. Presswood RG. Esthetics and color: perceiving the problem. Dent Clin North Am. 1977;21: 823-9.

37. Yap UJ, Ong G. Periodontal considerations in restorative dentistry. 1: Operative considerations. Dent Update 1994; 21:413-8.

38. Stein RS. Periodontal dictates for esthetic ceramometal crowns. J Am Dent Assoc 1987; (special issue):63E-73E.

39. Croll BM. Emergence profiles in natural tooth contour. Part II: clinical considerations. J Prosthet Dent 1990; 63: 374-9.

40. Reeves WG. Restorative margin placement and periodontal health. J Prosthet Dent 1991;6:733-6.

41. de Waal H, Castellucci G. The importance of restorative margin placement to the biologic width and periodontal health. Part II. Int J Periodontics Restorative Dent. 1994;14:70-83.
42. Hussaini S, Canela-Pichardo D. Palatal impression template for a fully edentulous arch during stage I implant placement. J Prosthet Dent 1997;77:630-2.

43. Tung FF, Nourbakhsh N, Bahn C. Accurate procedure for simultaneous registration of gingival emergence profile and maximal intercuspal position for metal ceramic restorations. J Prosthet Dent 2000;83:681-5.

44. Elian N, Tabourian G, Jalbout ZN, Classi A, Cho SC, Froum S, et al. Accurate transfer of periimplant soft tissue emergence profile from the provisional crown to the final prosthesis using an emergence profile cast. J Esthet Restor Dent 2007;19:306-14.

45. Attard N, Barzilay I. A modified impression technique for accurate registration of peri-implant soft tissues. J Can Dent Assoc. 2003; 69:80-3.

46. Macintosh DC, Sutherland M. Method for developing an optimal emergence profile using heat-polymerized provisional restorations for single-tooth implant-supported restorations. J Prosthet Dent 2004; 91:289-92.

47. Shor A, Schuler R, Goto Y. Indirect implantsupported fixed provisional restoration in the esthetic zone: fabrication technique and treatment workflow. J Esthet Restor Dent 2008; 20:82-95.

48. Silness J, Löe H. Periodontal disease in pregnancy 3. Response to local treatment. Acta Odontol Scand 1966;24:747-59.

49. Loe H, Silness J. Periodontal disease in pregnancy 1. Prevalence and severity. Acta Odontol Scand 1963;21:533-51.

\section{CORRESPONDENCIA}

Carlos Martín Ardila Medina

Carrera 47 No. 20 sur 46 Envigado Antioquía

57(4) 3348122

cmartin@odontologia.udea.edu.co 